\title{
SPLIT AND MINIMAL ABELIAN EXTENSIONS OF FINITE GROUPS $\left({ }^{1}\right)$
}

\author{
BY
}

VICTOR E. HILL

\begin{abstract}
Criteria for an abelian extension of a group to split are given in terms of a Sylow decomposition of the kernel and of normal series for the Sylow subgroups. An extension is minimal if only the entire extension is carried onto the given group by the canonical homomorphism. Various basic results on minimal extensions are given, and the structure question is related to the case of irreducible kernels of prime exponent. It is proved that an irreducible modular representation of $\operatorname{SL}(2, p)$ or $\operatorname{PSL}(2, p)$ for $p$ prime and $>5$ afford a minimal extension with kernel of exponent $p$ only when the representation has degree 3 , i.e., when the kernel has order $p^{3}$.
\end{abstract}

1. Introduction. Schur proved in 1911 that the central extensions of the unimodular group SL $\left(2, p^{n}\right.$ ) are all split (see [8]). Ashby Foote showed [4] that for an arbitrary finite group $G$ which coincides with its own derived group, there is a central extension $U$ of $G$ which is universal in the sense that $U$ admits no nonsplit central extension. In the special case $G \cong \operatorname{SL}\left(k, p^{n}\right), U$ coincides with $G$. No abelian extension with the property of Foote's central extension can exist because Gaschütz proved [5] that every finite group has a nonsplit abelian extension. Consequently, a useful question is how the existing nonsplit extensions may be classified.

The extensions farthest from splitting are those that $\mathrm{H}$. Wielandt has called minimal, in which the only subgroup of $E$ in the sequence

$$
1 \rightarrow A \rightarrow E \rightarrow G \rightarrow 1
$$

carried onto $G$ by the canonical homomorphism is $E$ itself. In this paper we show that in the special cases of $\operatorname{SL}(2, p)$ and $\operatorname{PSL}(2, p)$, if a minimal extension has an abelian kernel of exponent $p$ which is also an irreducible $K G$-module ( $K$ the field of order $p$ ), that kernel must have order $p^{3}$. We also prove results on split and minimal extensions which, apart from any intrinsic interest, suggest why minimal extensions with elementary abelian kernels may be singled out for study.

Received by the editors September 22, 1971.

AMS (MOS) subject classifications (1970). Primary 20F 25, 20G40; Secondary 20C20, $20 \mathrm{~K} 35$.

Key words and phrases. Modular representations, projective modules, block of defect zero, linear groups over finite fields, generators and relations.

( $\left.{ }^{1}\right)$ Most of these results were included in the author's $\mathrm{Ph}$. D. thesis written under the direction of Professor Charles W. Curtis at the University of Oregon.

Copyright (๑ 1973, American Mathematical Society 
We adopt the terminology that, in (1.1), $E$ is an extension of $G$ by $A, E$ is central if $A$ is in the center of $E$, and $E$ is abelian if $A$ is abelian. (This language conforms to that of many authors but differs, for example, from that of M. Hall [7].) If $A$ is abelian, then $E$ gives rise to a well-known homomorphism $\chi$ from $G$ into the automorphism group of $A$ such that

$$
\chi(g): a \rightarrow a^{g}=\left(g^{\prime}\right)^{-1} a g^{\prime}
$$

where the $g^{\prime}$ come from a complete set of coset representatives for $A$ in $E$, with each $g^{\prime}$ corresponding to $g \in G$ and $1^{\prime}$ to $1 \in G$.

All groups will be assumed finite unless specified otherwise. The letters $E$, $G, A$ will be reserved for groups with $E$ an extension of $G$ by $A$, and $K$ or $K_{j}$ will always denote the field of prime order $p$ or $p_{j}$, respectively. Following Wielandt and others, the following notations will be used:

$$
\begin{aligned}
& C \leq B \quad C \text { is a subgroup of } B, \\
& C<B \quad C \text { is a proper subgroup of } B, \\
& C \unlhd B \quad C \text { is a normal subgroup of } B, \\
& C \triangleleft B \quad C \text { is a proper normal subgroup of } B, \\
& |C| \text { order of } C \text {, } \\
& B \times C \text { direct product of } B \text { and } C, \\
& B \oplus C \text { direct sum of (additive) } B \text { and } C, \\
& \langle x, y, \ldots\rangle \text { group generated by }\{x, y, \ldots\} .
\end{aligned}
$$

In particular, if $E$ is an abelian extension, $\chi$ denotes the homomorphism (1.2), which makes $A$ a $G$-module. Now

(1.3) A subgroup $B$ of $A$ is a $G$-submodule if and only if $B \unlhd E$.

It will be useful to consider $G$-modules $B / C$ where $C \leq B \leq A$ and $C, B \unlhd E$; a special case is $B=A$, in which $E / C$ is an extension of $G$ by $A / C$.

We shall use several times a remark due to Dedekind:

(1.4) Lemma. If $X$ and $Y$ are subsets of a group $H$, if $T \leq H$, and if $Y T \subseteq Y$, then $(X \cap Y) T=X T \cap Y T$. In particular, if $X, Y \leq H$, then $Y T \subseteq Y$ may be replaced by $T \leq Y$.

2. Split abelian extensions. We begin with two results relating the splitting of certain homomorphic images of an extension $E$ to the splitting of $E$ itself.

(2.1) Theorem. Let $E$ be an extension of $G$ by $A$, with $G$ and $A$ finite or infinite. Let $A=A_{1} \times \cdots \times A_{r}$ with $A_{i} \unlhd E$ for $1 \leq i \leq r$, and $A_{i}^{*}=\Pi_{j \neq i} A_{j}$. Then

(i) E splits over $A$ if and only if $E / A_{i}^{*}$ splits over $A / A_{i}^{*}$ for all $i=$ $1, \cdots, r$.

(ii) For any $r \geq 2, E$ splits over $A$ if and only if $E / A_{1}$ and $E / A_{2}$ split over $A / A_{1}$ and $A / A_{2}$, respectively.

Proof. (i) Let $E$ split over $A$, that is, suppose there is $G^{*} \leq E$ with $E=$ 
$G^{*} A$ and $G^{*} \cap A=1$. For fixed $i$, each $A_{i}^{*} \unlhd E, E / A_{i}^{*}$ is an extension of $G$ by $A / A_{i}^{*}$, and $G^{*} A_{i}^{*} \leq E$. Now by (1.4),

$$
\left(G^{*} A_{i}^{*} / A_{i}^{*}\right) \cdot\left(A / A_{i}^{*}\right)=G^{*} A / A_{i}^{*}=E / A_{i}^{*}
$$

and

$$
\left(G^{*} A_{i}^{*} / A_{i}^{*}\right) \cap\left(A / A_{i}^{*}\right)=\left(G^{*} \cap A\right) A_{i}^{*} / A_{i}^{*}=A_{i}^{*} / A_{i}^{*}
$$

Consequently, each $E / A_{i}^{*}$ splits over $A / A_{i}^{*}$.

For the converse, there are subgroups $G_{1}^{*}, \ldots, G^{*}$ of $E$ such that, for $1 \leq$ $i \leq r, A_{i}^{*} \leq G_{i}^{*},\left(G_{i}^{*} A\right) / A_{i}^{*}=E / A_{i}^{*}$, and $\left(G_{i}^{*} / A_{i}^{*}\right) \cap\left(A / A_{i}^{*}\right)=A_{i}^{*} / A_{i}^{*}$. Then $G_{i}^{*} A$ $=E$ and $G_{i}^{*} \cap A=A_{i}^{*}$, for each $i$. Let $G^{*}=G_{1}^{*} \cap \ldots \cap G_{r}^{*}$. Then by $r$ applications of (1.4) with the observation $A_{i}^{*} \leq G_{i}^{*}$,

$$
\begin{aligned}
G^{*} A & =G^{*}\left(A_{1} A_{1}^{*}\right)=\left(G_{2}^{*} \cap \ldots \cap G_{r}^{*}\right) A_{2}\left(A_{3} \cdots A_{r}\right) \\
& =\cdots=G_{r}^{*} A_{r}=E,
\end{aligned}
$$

and

$$
\begin{aligned}
G^{*} \cap A & =\left(G_{1}^{*} \cap \ldots \cap G_{r-1}^{*}\right) \cap A_{r}^{*} \\
& =\cdots=G_{1}^{*} \cap\left(A_{2}^{*} \cap \ldots \cap A_{r}^{*}\right)=1 .
\end{aligned}
$$

(ii) First, let $E=G^{*} A$ with $G^{*} \cap A=1$. Then for $j=1,2$,

$$
\left(G^{*} A_{j} / A_{j}\right) \cdot\left(A / A_{j}\right)=G^{*} A_{j} A / A_{j}=E / A_{j}
$$

and, by (1.4),

$$
\left(G^{*} A_{j} / A_{j}\right) \cap\left(A / A_{j}\right)=\left(G^{*} \cap A\right) A_{j} / A_{j}=A_{j} / A_{j} ;
$$

therefore, $E / A_{1}$ and $E / A_{2}$ split.

Conversely, let $E / A_{j}=G_{j}^{*} A / A_{j}$ with $G_{j}^{*} \cap A=A_{j}$, for $j=1,2$. Let $B=$ $G_{1}^{*} A_{3} \cdots A_{r} ;$ then $B \leq E$ and $B A_{2}=E$ since $A_{1} \leq G_{1}$ and $A$ is a direct product. Now let $G^{*}=B \cap G_{2}^{*}$. Then $G^{*} A=E$ by (1.4) together with $B A_{2}=E$, and $G^{*} \cap A=B \cap A_{2}$. Now if $x \in B \cap A_{2}$, then $x=b a$ for some $b \in G_{1}^{*}$ and $a \epsilon$ $A_{3} \cdots A_{r}$ (by definition of $B$ ), and $b a \in A_{2}$. Hence $b \in A$ and thus $b \in A_{1}$. Consequently, $b a \in A_{1}\left(A_{3} \cdots A_{r}\right) \cap A_{2}=1$, and $x=1$, so $E$ is split.

(2.2) Corollary. Let $E$ be an extension of a finite or infinite group $G$ by $a$ finite nilpotent group $A$, let $A=A_{1} \times \cdots \times A_{r}$ be a Sylow decomposition of $A$, and let $A_{i}^{*}=\Pi_{j \neq i} A_{j}$ for $i=1, \cdots$, r. Then

(i) E splits over $A$ if and only if $E / A_{i}^{*}$ splits over $A / A_{i}^{*}$ for all $i=$ $1, \cdots, r$;

(ii) $E$ splits over $A$ if and only if $E / A_{j}$ splits over $A / A_{j}$ for two distinct $j \in\{1, \cdots, r\}$. 
Proof. The result follows immediately from (2.1) since a nilpotent group has a Sylow decomposition of the required form, where the $A_{i}$ are characteristic subgroups of $A$.

This corollary suggests that splitting of abelian extensions may be approached through splitting of extensions by the Sylow subgroups of the kernels (where $\left.A / A_{i}^{*} \cong A_{i}\right)$. The next result shows that abelian kernels of prime exponent $p$ and irreducible representations over the field $K$ of order $p$ are appropriate to be singled out for study.

(2.3) Proposition. Let $E$ be an extension of a (possibly infinite) group $G$ by a finite abelian p-group $A$, let $\chi$ be as in (1.2), and let

$$
A=A_{0} \triangleright A_{1} \triangleright \cdots \triangleright A_{r}=1
$$

be a $X$-composition series for $A$ (in the sense of (1.3)). Then each factor $A_{i-1} / A_{i}(1 \leq i \leq r)$ is an abelian group of exponent $p$, affording an irreducible representation of $G$ over the $K=\mathrm{GF}(p)$.

Proof. Let $|A|=p^{n}$. Then $A$ has a subgroup of order $p^{n-1}$, and the corresponding factor group is elementary abelian. Since $A$ is finite, it has a proper normal subgroup $A^{*}$ of smallest possible order such that $A / A^{*}$ is elementary. It is easily shown that $A^{*}$ is contained in every other subgroup of $A$ yielding an elementary factor group and, moreover, that $A^{*}$ is a characteristic subgroup of $A$. Hence $A^{*} \unlhd E$ and, by (1.3), $A^{*}$ is $\chi$-invariant. Repeating this argument yields a series

$$
A \triangleright A^{*} \triangleright A^{* *} \triangleright \cdots \triangleright 1
$$

consisting of subgroups of $A$ invariant under $\chi$, which series can be refined to a $\chi$-composition series for $A$ in which each factor group is elementary abelian.

Now $\chi$ acts on $A_{i-1} / A_{i}$ by restriction, and if $A_{i-1} / A_{i}$ is written additively as a $K$-module, then $\chi$ is a representation of $G$ over $K$ afforded by the $K G$ module $A_{i-1} / A_{i}$. If this module were $\chi$-reducible there would be a $\chi$-invariant subgroup $C / A_{i}$ which, by (1.3), would introduce a new term into the series (2.4). Hence $A_{i-1} / A_{i}$ affords an irreducible $K$-representation, and the proof of (2.3) is complete.

Arguments similar to those in the proof of (2.1) yield

(2.5) Let $E$ be an extension of $G$ by $A$ (with $G$ and $A$ finite or infinite). If $A$ has a series

$$
A=A_{0} \triangleright \cdots \triangleright A_{r}=1
$$

such that $A_{i} \unlhd E$ for each $i=0,1, \ldots, r$, and if there are subgroups $B_{1}, \cdots$, $B_{r}$ of $E$ such that $E=B_{1} A$ and $B_{1} \cap A=A_{1}$ and, for each $i, B_{i}=B_{i+1} A_{i}$ and $B_{i+1} \cap A_{i}=A_{i+1}$, then $E$ splits as $B_{r} A$.

Now the proof of a theorem of Marshall Hall may be applied to the special 
case of elementary abelian kernels to yield

(2.6) Theorem (see [7, Theorem 15.5.1]). If $E$ is an extension of $G$ by $A$, $A$ is an abelian group of prime exponent $p, \chi$ is as in (1.2), and $A$ is a free $K G-$ module affording $\chi$, then $E$ is split.

This result may be generalized by (2.5) to obtain the following stronger result:

(2.7) Theorem. If $E$ is an extension of $G$ by an abelian p-group $A$ with $\chi$ : $G \rightarrow$ Aut $(A)$ as in (1.2), and if $A=A_{0} \triangleright \cdots \triangleright A_{r}=1$ is a $\chi$-normal series for $A$ such that each factor is a free KG-module, then $E$ is split.

Proof. Since $A_{i} \unlhd E$ (from the proof of (2.3)) $E / A_{1}$ is an extension of $G$ by $A / A_{1}$, where $A / A_{1}$ affords a restriction of $\chi$. By the hypothesis together with (2.6), $E / A_{1}$ splits as $\left(B_{1} / A_{1}\right)\left(A / A_{1}\right)$. The same argument applies to $A_{2} \unlhd B_{1}$ and the module $A_{1} / A_{2}$. Repetitions yield $B_{1}, \cdots, B_{r}$ satisfying the hypotheses of (2.5).

A principal result of this section is

(2.8) Theorem. Let $E$ be an extension of $G$ by an abelian group $A$, let $\chi$ : $G \rightarrow$ Aut $(A)$ as in (1.2), and let $A=A^{1} \times \cdots \times A^{s}$ be a Sylow decomposition of $A$, where $A^{j}$ corresponds to the prime $p_{j}, 1 \leq j \leq s$. If for each $p_{j}$ dividing $|G|$, each factor of some $\chi$-normal series for $A^{j}$ is a projective $K_{j} G$-module, then $E$ is split.

Proof. We consider first the special case in which $A$ is an abelian $p$-group, that is, $s=1$. Let $A=A_{0} \triangleright A_{1} \triangleright \cdots \triangleright A_{r}=1$ be a $\chi$-normal series with projective factors, and let $X$ be an arbitrary Sylow p-subgroup of $G$. Since $A_{i-1} / A_{i}$ is projective, it is a direct sum of principal indecomposable modules of $G$, each of which, when viewed as a $K X$-module, is isomorphic to a direct sum of copies of the (right) regular $K X$-module $K X$ (by a theorem of Green [6], see [3, (65.16)]). Hence $A_{i-1} / A_{i}$ is isomorphic to a direct sum of copies of the regular $K X$-module and so is a free $K X$-module, for $1 \leq i \leq r$. Now let $X^{*}$ be the restriction of $E$ to $X$ (i.e., $X^{*}$ is the subgroup of $E$ corresponding to $X$ under the canonical homomorphism); then $X^{*}$ is an extension of $X$ by $A$, and $A$ affords the restriction of $\chi$ to $X$. By (2.7), $X^{*}$ splits over $A$. But $p$ is the only prime dividing both $|A|$ and $|G|$. Moreover, by the Schur-Zassenhaus Theorem we have the fact that, for every prime $q \neq p$ dividing $|G|$, the restriction of $E$ to some Sylow $q$-subgroup $Q$ of $G$ splits when regarded as an extension of $Q$ by $A$. Hence (see [7, Theorem 15.8.6]) $E$ splits over $A$.

To complete the proof of (2.8), let $A_{*}^{j}=\Pi_{k \neq j} A^{k}$. By (2.1), E splits if and only if $E / A_{*}^{j}$ splits over $A / A_{*}^{j}$ for $j=1, \ldots, s$. Now $A / A_{*}^{j}$ is $\chi$-isomorphic to $A^{j}$, so $E / A_{*}^{j}$ is an extension of $G$ by an abelian $p_{j}$-group. If $p_{j}$ divides $|G|$. 
then $E / A_{*}^{j}$ splits by the special case above because the property of being a projective $K_{j} G$-module is carried over from the $\chi$-normal factors of $A^{j}$ to those of $A / A_{*}^{j}$ by $\chi$-isomorphism. If $p_{j}$ does not divide $|G|$, then $E / A_{*}^{j}$ splits by the Schur-Zassenhaus Theorem. Therefore, $E$ splits.

In the terminology of modular representation theorv, we have

(2.9) Corollary. Let $E$ be an extension of $G$ by an abelian group $A$, let $X$ be as in (1.2), and let $A=A^{1} \times \cdots \times A^{s}$ be a Sylow decomposition of $A$, where $A^{j}$ corresponds to the prime $p_{j}, 1 \leq j \leq s$. If for each $p_{j}$ dividing $|G|$, each factor of some $\chi$-normal series for $A^{j}$, viewed as a $K_{j} G$-module, belongs to a block of defect 0 , then $E$ is split.

Proof. By a result of Brauer and Nesbitt [2] (see [3, (86.3)]), each factor of the $\chi$-normal series is a principal indecomposable $K_{j} G$-module. Hence by $[3,(56.6)]$ each factor is projective. Thus if $p_{j}$ divides $|G|$, then $E / A_{*}^{j}$ splits, and with this one modification the proof of (2.8) applies.

This corollary shows that the application of modular representation theory to finding nonsplit abelian extensions entails the use of modules from blocks of defect greater than zero. If $E$ is an abelian extension of $G$ by $A$ which does not split, then $A$ must have a Sylow $p$-subgroup $A^{j}$ for some $p_{j}$ dividing $|G|$ such that $A^{j}$ has a $\chi$-normal factor which (viewed as a $K_{j} G$-module) belongs to a block of defect greater than zero.

3. Minimal abelian extensions. Let $E$ be an extension of $G$ by $A$ (with $G$ and $A$ finite or infinite) and $\pi: E \rightarrow G$ the canonical homomorphism. Helmut Wielandt (in some unpublished work: see footnote 4 of [5]) has called $E$ minimal if whenever $T \leq G$ and $\pi(T)=G$, it follows that $T=E$.

Equivalently, $E$ is minimal if and only if $T \leq E$ with $T A=E$ implies $T=E$. Thus minimality may be regarded as the strongest sort of nonsplitting. Another useful characterization is

(3.1) $E$ is a minimal extension of $G$ by $A$ if and only if $A$ is contained in the Frattini subgroup of $E$.

This result was proved by $H$. Bechtell in [1] and was used earlier by Gaschütz in [5].

That not every nonsplit extension is minimal is clear from the quaternion group of order 8 , regarded as an extension of a cyclic group of order 2 by one of order 4. However, under certain conditions it is true that every nonsplit extension is minimal. For example, the following result will be useful later:

(3.2) If $E$ is an extension of $G$ by an abelian group $A$ of prime exponent $p$, and if $A$ is an irreducible $K G$-module affording $\chi: G \rightarrow$ Aut $(A)$, then $E$ is either split or minimal. 
Proof. If $E$ is not minimal, then there is $T<E$ such that $T A=E$. Now $T \cap A<A$ since $A$ is not contained in $T$. Moreover, $T \cap A \unlhd T$ and $T \cap A \unlhd A$ since $A$ is abelian. Hence $T \cap A \unlhd E$ and, by (1.3), $T \cap A$ is a proper $K G$-submodule of $A$. But $A$ is irreducible, so $E$ is split.

Three easy consequences are

(3.3) If $E$ is an extension of a (possibly infinite) group $G$ by a cyclic group of prime order, then either $E$ is split or $E$ is minimal.

(3.4) The only minimal extension of a finite cyclic group $G$ is again a cyclic group.

(3.5) Let $E$ be a minimal extension of $G$ by $A$ (with $G$ and $A$ finite or infinite), and let $B \leq A$ with $B \unlhd E$. Then $E / B$ is a minimal extension of $G$ by $A / B$.

Proof. The first result follows immediately from (3.2). For the second, let $G=\left\langle g: g^{n}=1\right\rangle$ and let $E$ be a minimal extension of $G$ by a finite $A$. Let $g^{*}$ be a representative in $E$ for the coset corresponding to $g$ under the canonical homomorphism $\pi$. Then $\pi$ carries $T=\left\langle g^{*}\right\rangle$ onto $G$, and $T=E$. For (3.5), if $E / B$ is not minimal, then there exists $T<E$ with $B \leq T$ such that $(T / B)(A / B)$ $=E / B$. But then $T A / B=E / B$ and $T A=E$, which contradicts the minimality of $E$.

Gaschütz has proved [5] that an arbitrary finite group $G$ has a minimal extension $H^{(p)}$ of largest possible (finite) order such that the kernel $H_{p}$ is abelian of exponent $p$ ( $p$ a prime dividing $|G|)$ and that $H^{(p)}$ is unique up to equivalence of extensions. Let $B_{p}$ be a maximal proper $K G$-submodule of $H_{p}$; then $H_{p} / B_{p}$ is an irreducible $K G$-module, and by (3.5), $H^{(p)} / B_{p}$ is a minimal extension of $G$ by $H_{p} / B_{p}$. In fact, if $E$ is any minimal extension of $G$ by an abelian group $A$ of exponent $p$, and if $B$ is a maximal proper $K G$-submodule of $A$, then $E / B$ is a minimal extension of $G$ by $A / B$, and $A / B$ is an irreducible $K G$-module. Consequently, a knowledge of the minimal extensions of $G$ with irreducible kernels of exponent $p$ is important for the investigation of minimal extensions.

(3.6) Theorem. Let $p$ be a prime $\geq 5$ and let $G \cong \operatorname{SL}(2, p)$ or $\operatorname{PSL}(2, p)$. If an irreducible modular representation of $G$ over $K=\mathrm{GF}(p)$ affords a minimal extension of $G$, then the representation bas $K$-degree 3 . Hence, if $E$ is a minimal extension of $G$ by a finite abelian group $A$ of exponent $p$, then $A$ is of order $p^{3}$.

Proof.(2) Let $A$ be an irreducible $K G$-module and $G \cong \operatorname{SL}(2, p)$. If $A$ is faithful, then $E$ is split since, if $C$ is the centralizer in $E$ of a coset representative corresponding to the central element of $G$, then $E=C A$ and $C \cap A$ $=1$. Hence we may assume that $A$ is not faithful or that $G \cong \operatorname{PSL}(2, p)$; in either event, $A$ is a $\operatorname{PSL}(2, p)$ module, and thus we may assume $G \cong \operatorname{PSL}(2, p)$.

$\left.{ }^{2}\right)$ The author expresses his appreciation to the referee, who provided this shorter method of proof. 
By (3.2), it suffices to prove that if the degree $k$ of $A$ is not 3, then $E$ splits over $A$.

Let $x^{*}$ be an element of order $p$ in $G$, and let $y^{*} \in G$ be of order $(p-1) / 2$ with $y^{*-1} x^{*} y^{*}=x^{* \lambda}$, where $\lambda$ is a primitive $(p-1) / 2$ th root of 1 in $K$. Brauer and Nesbitt proved [2] that $\operatorname{dim} A=2 m+1 \leq p$ for some integer $m$, and $A$ has a basis $\left\{a_{i}:-m \leq i \leq m\right\}$ with $a_{i} y^{*}=\lambda^{i} a_{i}$. Moreover, $a_{m}$ spans the unique one-dimensional subspace of $A$ which is invariant under the action of $x^{*}$. If $\operatorname{dim} A=p$, then by [2], $A$ is projective, and, by (2.8), $E$ is split. Hence we have $m<$ $(p-1) / 2$. A calculation performed on the representations in [2] shows that, for such $m$ and for any $a \in A$,

$$
\sum_{i=1}^{p} a x^{* \lambda i}=0 .
$$

Let $x$ be a $p$-element of $E$ which maps to $x^{*} \in G$ and $y$ be an element of order $(p-1) / 2$ which maps to $y^{*}$. If we write $A$ multiplicatively, the relations of the preceding paragraph become

$$
y^{-1} a_{i} y=a_{i}^{\lambda i}, \quad x^{-1} a_{m} x=a_{m},
$$

and $\left\langle a_{m}\right\rangle$ is the center of the Sylow $p$-subgroup of $E$ containing $x$. Hence there is some $c \in K$ such that

$$
x^{p}=a_{m}^{c}
$$

Moreover, if $a \in A$, then $\Pi_{i=1}^{p} x^{-\lambda i} a x^{\lambda i}=1$, and $y^{-1} x y=x^{\lambda} b$ for some $b \in A$. Hence

$$
\begin{aligned}
y^{-1} x^{p} y & =\left(y^{-1} x y\right)^{p}=\left(x^{\lambda} b\right)^{p}=x^{p \lambda} \prod_{i=1}^{p} x^{-\lambda i} b x^{\lambda i} \\
& =a_{m}^{c \lambda} \quad \text { by (3.7) and (3.8). }
\end{aligned}
$$

But then

$$
a_{m}^{c \lambda}=y^{-1} x^{p} y=y^{-1} a_{m}^{c} y=a_{m}^{c \lambda}
$$

so $c\left(\lambda-\lambda^{m}\right)=0$ in $K$. Thus either $c=0$ or $\lambda=\lambda^{m}$. If $c=0$, then $x^{p}=1$ and the Sylow $p$-subgroup of $E$ containing $x$ splits over $A$. But then (see [7, Theorem 15.8.6]) $E$ splits over $A$. Hence $\lambda=\lambda^{m}$ and $m=1$, so $\operatorname{dim} A=2 m+1=3$.

The question of whether $\operatorname{SL}(2, p)$ or $\operatorname{PSL}(2, p)$ has more than one inequivalent minimal extension by an abelian group of type $(p, p, p)$ has not yet been answered.

\section{REFERENCES}

1. H. F. Bechtell, Reduced partial products, Amer. Math. Monthly 72 (1965), 881-882. MR $32 \sharp 4185$.

2. R. Brauer and C. Nesbitt, On the modular characters of groups, Ann. of Math. (2) 42 (1941), 556-590. MR 2, 309. 
3. C. W. Curtis and I. Riener, Representation theory of finite groups and as sociative algebras, Pure and Appl. Math., vol. XI, Interscience, New York, 1962. MR $26 \# 2519$.

4. S. A. Foote, Universal central extensions of groups, $\mathrm{Ph}$. D. Thesis, University of Wisconsin, Madison, Wis., 1965.

5. W. Gaschütz, Uber modulare Darstellungen endlicher Gruppen, die von freien Gruppen induz iert werden, Math. Z. 60 (1954), 274-286. MR 16, 446.

6. J. A. Green, $O_{n}$ the indecomposable representations of a finite group, Math. Z. 70 (1958/59), 430-445. MR 24 \#A1304.

7. M. Hall, Jr., The theory of groups, Macmillan, New York, 1959. MR 21 \#1996.

8. I. Schur, Über die Darstellungen der symmetrischen und der alternierenden Gruppen durch gebrochene lineare Substitutionen, J. Reine Angew. Math. 139(1911), 155-250.

DEPARTMENT OF MATHEMATICS, WILLIAMS COLLEGE, WILLIAMSTOWN, MASSACHUSETTS 01267 